\title{
PPE Misuse and its Effect on Infectious Disease Among EMS in Saudi Arabia Abdulaziz Alshammari ${ }^{\mathrm{a}}$, Jean B. Bail ${ }^{\mathrm{a}}$, Steven J. Parillo ${ }^{\mathrm{a}}$
}

Emergency Medical Services (EMS) responders provide medical services in diverse, unique and dangerous environments. EMS provides care to diverse and mobile populations who are likely to contract infectious diseases. In addition to responding to the emergency situations, the EMS are also attending to the homeless, trauma victims and nursing home patients in addition to critically ill patients with diverse diseases and infections. Like most healthcare professionals, EMS responders are faced with increased exposure to infectious conditions. As a result, they are concerned with their safety and preventive measures against any infection. The aim of this study is to assess whether EMS follows the standard guidelines provided by the Saudi Red Crescent Authority (SRCA) to avoid being infected. Using the standard SRCA observational tool, EMS Red Crescent workers in various stations, EMS station 1 (17 paramedics), EMS station 2 (11 paramedics) and EMS station 3 (16 paramedics), were observed on whether they follow the provided standard Personal Protective Equipment (PPE) guidelines to avoid being infected whether by MERS or other diseases. The results indicate that most of the EMS staff did not implement the PPE standard procedure or failed to demonstrate the skills on the application of the standards. Failure to follow the safety standards and guidelines is the main cause of infections among the EMS staff (Disaster Med Public Health Preparedness. 2018). Therefore, there is need to provide comprehensive training on the standard guidelines and safety measures to prevent the occurrences of infections among the EMS workforce.

Keywords: PPE misuse, disaster medicine

EMS responders to pandemic influenza in Saudi Arabia

Our world is encompassed in life threatening manmade and natural scenarios that not only impact the lives of individual, but also the socio-politico and economic climate of a nation. With this in mind, several emergency services that function as "individual" services for the safety of the people is provided by the administrative bodies. These emergencies medical services (EMS) function as managing, supervising and coordinating as well as individual assisting units at the time of an emergency (Bekefi, T., Epstein M. J., \& Yuthas, K. 2008). Emergency medical services providers and agencies alike participate in life-saving activities every day. Workers in these agencies provide assistance in daily emergency situations, transporting, and treating patients in various situations. Their input in the medical process is essential as they ensure the welfare of the patient.

The healthcare sector is exposed to varying infections. (NIOSH. 2017). despite the availability of multiple protection procedures. Contamination remains a significant issue to the Emergency medical services responders as ambulances become a constant source of various pathogenic bacteria and viruses as a result of transporting patients from accident scenes and other emergency medical issues to the medical facilities. The transportation procedure creates a situation where the paramedics as well as the patient are exposed to the pathogens, a majority of which may be infectious (Reed, et al., 1993). Although there are various safeguards and disposable personal protective medical equipment that reduce the risk to the medical staff, the transportation process remains susceptible to contamination from blood and infectious substance. The result of these infections on the EMS in Saudi Arabia specifically is significant, as more than 35 medical providers have been identified as infected with MERS (Ministry of health, coronavirus, 2018).

The ambulance service managed by the Saudi Red Crescent Authority (SRCA) is the focus for this project for its review of policy and guidelines on the pre-hospital process and the current policies regarding to reduce infections. Precautions such as disinfection and sterilization of ambulances and equipment are not accompanied by corresponding work practice and PPE in many cases (Haggman, Kenkre, \& Wallace, 2016). SRCA is a humanitarian society founded in 1935 that has 384 ambulatory centers and there are over 8, 000 EMS staff using approximately 2,000 ambulances, and they are all at risk (SRCA). To ensure effective control of possible infections, a significantly holistic approach is required; one which is evidence-based and also cost-effective.

\section{Background}

Inadequate policies and interventions or personal failures of the emergency medical services team are a facilitator for infections among the healthcare workers. Thus, the policies and practices need to be identified which can prevent such infections. Meanwhile, the lack of standardization attributed to poor intervention and training practices, lack of policies and other governing challenges have led to the poor control of infectious disease within the emergency medical services team (Al-Shammari et al., 2017). This disparity has further been associated in view of the educational discrepancy and varying core values and competencies of the training institutes for this collective body, because of which, there is an inconsistency in the practice measures amongst these workers (Al-Shammari et al., 2017).

As it has been recognized that the poor education and training is an indicator of poor standards and often a facilitator of increased infections among the healthcare professionals. As a result of which in many contexts the EMS in Saudi Arabia which is primarily focused on the transferring and transportation of the patients, can be a driver of infection and increased fatality for the patient, owing to the lack of training and standardization amongst the same (Alnazi et al. 2014). 
The requirement for disaster medicine is more crucial than ever before with diseases such as MERS affecting areas deemed disaster-prone. Medical workers and emergency service providers at large require evidence as a stepping stone to new decisions and measures involving medical care for health workers. EMS in Saudi Arabia has faced its fair share of challenges, with a number of its employees contracting the deadly virus MERS (Gushulak, 2017). Red Crescent plans to use this information to creating effective influenza plans which will enhance its existing operational protocols.

In particular, command and control plans will require thorough assessment which will benefit all parties involved. According to Costa et al. (2015), interventions such as PPE should be prioritized before traveling to a crowded area (Makkah, Jeddah and Madina) ensure that volunteers and medical professionals going on a mission are adequately considered in regard to their health. The project used observation as the tool. Information was derived by working with the EMS employees and observing them to assess their response to the issues of workplace infections. The study already got permission from the Red Crescent to speak to its employees on how the gap can be bridged (Appendix B). The study once carried out will be useful to both the authorities and the employees. The employees will have proper medical care, while the authority will benefit from the job security provided by the employees. The organizational structures will also support the humanitarian aspect while securing the fair decisions in the daily operations of the workers.

\section{Literature Review}

The importance of adequate policy guidelines in preventing infection of healthcare workers is inarguable, with benefits such as better individual health. Costa et al. (2015) focused on reviewing existing information on the delivering interventions before the workers were deployed to their missions. They aimed to understand the benefits of the intervention. Majority of the identified results contended that workplace health is crucial to the success of the lifesaving efforts of the employees. Just as in the case of the EMS from Red Crescent, the policies did not place emphasis on the workplace assessments. The study discovered that the workers were exposed to high-risk infections which included TB, influenza, HIV, and other dangerous infections (Costa, et al. 2015). These infections depended on the region, but the results are a clear indication that the pre-mission assessment that include standard precautions policy should be mandatory.

Effective policy is significant in identifying and managing the risk posed by these diseases to the workers. For any health care worker, especially those in humanitarian crises, bloodborne pathogens may be transmitted through exposure to the infected patients. It could either be percutaneous or mucosal, with the risk of exposure ranging between $0.3 \%$ to $30 \%$ in cases where the worker is exposed to HIV and HBV respectively. According to Beltrami, Williams, Shapiro, and Chamberland (2010), only a sustained obligation to the health care workers will provide the prerequisite protection they require. Due to the risky aspect of their occupations, the threat of exposure is ever present (Beltrami et al., 2010). The authors call for adherence to disease control regulations on dealing with the threats of infectious diseases by providing vaccines, creating exposure control plans, and ensuring frequent training of their employees. Hence, the need arises to ensure continuous improvement of definitions of exposure, the effectiveness of the preventive procedures, as well as sustained commitment to the overall health of medical workers.

Recent rises in the number of infectious diseases in humanitarian crises led to an unwillingness by workers to take part in risky missions (Gee, S., \& Skovdal. (2017). One of the infections which have caused the apathy among emergency services workers is MERS. The infectious disease has spread in some of the areas which already face limited healthcare infrastructure. In their study, Haggman, Kenkre, and Wallace (2016) focus on reviewing the impact of Ebola in regard to the overall willingness of healthcare workers to respond to areas such as Liberia and Sierra Leone which are some of the most affected such nations.

Figures in recent studies show that in 2015, only two cases of Ebola were reported, but in the following $1 \frac{1 / 2}{2}$ years, more than 11,000 people died, including 400 medical workers. Their study also focused on Red Cross and Red Crescent employees and their issues with deployment to such regions (Haggman et al., 2016). The workers raised concerns about their overall welfare in such countries, while also highlighting the apprehensions cited by their close relatives. A major aspect underlining the issues they raised was the lack of adequate operating procedures to guarantee their quality of life. There was also no guarantee of their safety. This study highlights the changes posed by infectious diseases and why it is critical for service providers to enhance the health conditions of their workers. In addition to health, safety was also highlighted as a precondition for ensuring the occupational health of the professionals in these situations (Haggman et al., 2016). Hence, the need arose to create policies which will guarantee the two aspects highlighted by the healthcare workers.

Various methods of managing infectious diseases by the health workers have been discussed. Koenig (2014) stresses various approaches to be utilized by EMS to limit their exposure. A critical policy proposed is the identify, isolate, and inform policy. The emergency services workers already have training on the signs of an infectious disease, which should be applied in this instance. They should then isolate the risk, which will reduce their exposure and others to the deadly infections. The last step involves using the protocol in place to provide the information to fellow workers and relevant authorities (Koenig, 2014). Due to the direct threat posed by the infections, the author provides these screening methods to service providers to protect themselves, while also protecting the patients. The methods applied in this study stress the fact workers should also take the initiative to protect themselves (Koenig, 2014). Due to the bureaucratic nature of this step, the EMS should ensure they put the details in their policies to ensure notifications of infectious diseases are dealt with before they affect the first responders.

The articles by various authors have raised the same concerns - the risks posed by infectious diseases to the humanitarian workers. The consensus is that EMS should do more to protect the workers from infection, by the implementation of various policy decisions. Despite the dangers posed by these diseases, the responsibility still lies with the Red Crescent and its workers. Hence, disaster disease management should include protecting the employees to ensure a better quality of life and overall satisfaction. 


\section{Methods}

The projects foundation was on the question of whether authorities, specifically, the EMS, played part in the workers contracting infectious diseases. Red Crescent's guidelines and procedures were assessed to note the specific impact they have on addressing risks faced by their employees. In addition, the polices were compared to the World Health Organization standardized checklist (Appendix A). The research was carried out in 2018 between February to April. Permission was obtained from the Red Crescent authority to conduct the research and to create recommendation for consideration (appendix C). The participants of the study were employees of the EMS. The authority is an emergency services provider whose employees are potentially exposed to infectious diseases during treatment and transport of victims on a daily basis. The employees in the study were field officers as well as office workers of various ranks. The assumption was that they were all exposed to infectious diseases or have been in contact with fellow employees who were infected. Diversity in the respondents was a consideration, to ensure different views on each observation made.

Observations were performed over a period of three weeks during all shifts and three stations to reach approximately $50 \%$ of the paramedics. Data were scored using the checklist for each call observed (Appendix A). Recommendations were developed to be present to the red crescent authority. The data were processed, classified, and tabulated, reducing it in terms of specificity. A graphical analysis included helping to understand the observation data collected. The new information was shared with the relevant parties, including the Red Crescent.

The current guidelines and procedures for EMS (Red Crescent) workers in Saudi Arabia do not adequately address potential risk associated with treating patients who suffer from infection diseases. Since 2012, a few EMS workers have contracted to Middle East Respiratory Syndrome, MERS (Ministry of Health, coronavirus, 2018). This research investigated whether infection in responders results from policy and guidelines or EMS personal failing to follow the guidelines. The results are intended to provide guidance to EMS agencies in Saudi Arabia in their pandemic influenza plans and operational protocols. The audience for this subject is EMS agencies (Red Crescent).

Red Crescent (EMS)

Hail City, Saudi Arabia

EMS station 1 (17 paramedics)

EMS station 2 (11 paramedics)

EMS station 3 (16 paramedics)

\begin{tabular}{lcccccccc}
\hline Saudi Red Cre scent Authority SRCA & & & & & & \\
\hline Day; 8:00 & Night; 22:00 & & & & & & \\
\hline & Sat & Sun & Mon & Tue & Wen & Thu & Fri \\
\hline Station 1 & Day & Night & Off & Day & Day & Off & Off \\
\hline Station 2 & Day & Night & Off & Day & Day & Off & Off \\
\hline Station 3 & Day & Night & Off & Day & Day & Off & Off \\
\hline & & & & & & & \\
\hline
\end{tabular}

(EMS) providers play an important role in the prevention and control of infections. EMS providers are at the front line of medical care and have a high risk of exposure to patients with known or unknown infectious diseases.

Using the standard observational tool on EMS Red Crescent workers in various stations, EMS station 1 (17 paramedics), EMS station 2 (11 paramedics) and EMS station 3 (16 paramedics) were focused on seven key strategies as infection control measures in the EMS:

Use of personal protective equipment, e.g. gowns, gloves, eye, face and respiratory protection Hand Hygiene

Environmental decontamination and disinfection

Source control measures aimed at containing the patient's secretions Limiting contact with patients

Ambulance cleaning and disinfection.

Knowledge of EMS workers about infection prevention and control standards.

\begin{tabular}{|c|c|c|c|c|}
\hline \multirow[t]{2}{*}{ Results } & & $\mathrm{N} / \mathrm{A}$ & F ail & \multirow{2}{*}{$\begin{array}{r}\text { Pass } \\
3\end{array}$} \\
\hline & & 1 & 2 & \\
\hline DONNING - & PERSONAL ITEMS & 21 & 0 & 21 \\
\hline DONNING - & SHOES COVERS & 42 & 0 & 0 \\
\hline DONNING - & GOWN & 27 & 1 & 14 \\
\hline DONNING - & MASK & 0 & 8 & 34 \\
\hline DONNING - & FACE SHIELD & 16 & 3 & 23 \\
\hline DONNING - & HAIR COVER & 42 & 0 & 0 \\
\hline DONNING - & SANITZE HANDS & 42 & 0 & 0 \\
\hline DONNING - & GLOVES & 0 & 0 & 42 \\
\hline DOFFING - & GLOVES & 0 & 17 & 25 \\
\hline DOFFING - & Gown & 27 & 8 & 7 \\
\hline DOFFING - & SHOES COVERS & 42 & 0 & 0 \\
\hline DOFFING - & HAND HY GIENE & 35 & 4 & 3 \\
\hline DOFFING - & FACE SHIELD & 17 & 3 & 22 \\
\hline DOFFING - & HAIR COVER & 42 & 0 & 0 \\
\hline DOFFING - & MASK & 0 & 17 & 25 \\
\hline DOFFING - & HAND HYGIENE & 0 & 10 & 32 \\
\hline
\end{tabular}

N/A: The skill was indicated, but not performed

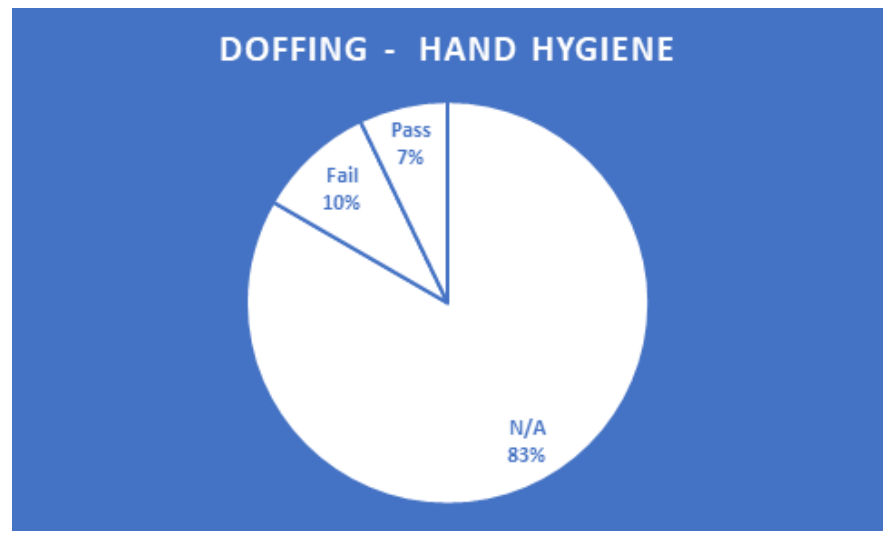

Figure 1. In the doffing part of Hand Hygiene, seen that $83 \%$ did not perform the skill when needed, $7 \%$ successful demonstrated proper technique and $10 \%$ failed to perform proper technique. 


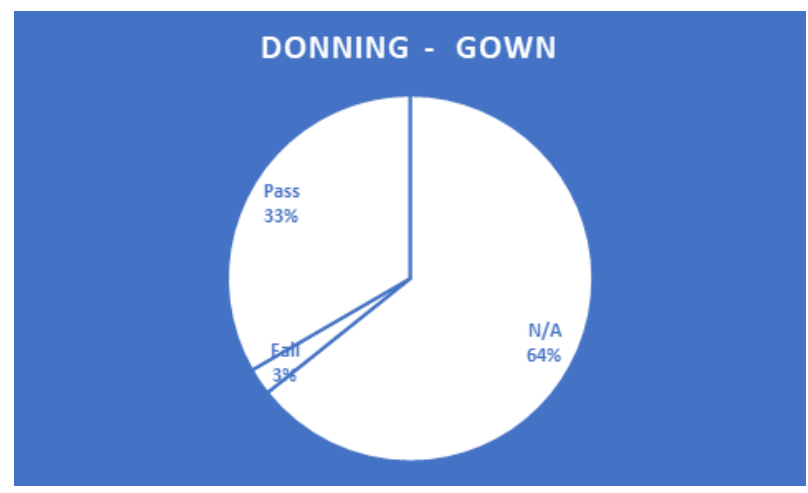

Figure 2. In the donning part of Gown, seen that $64 \%$ did not perform the skill when needed, $33 \%$ successful demonstrated proper technique and $3 \%$ failed to perform proper technique.

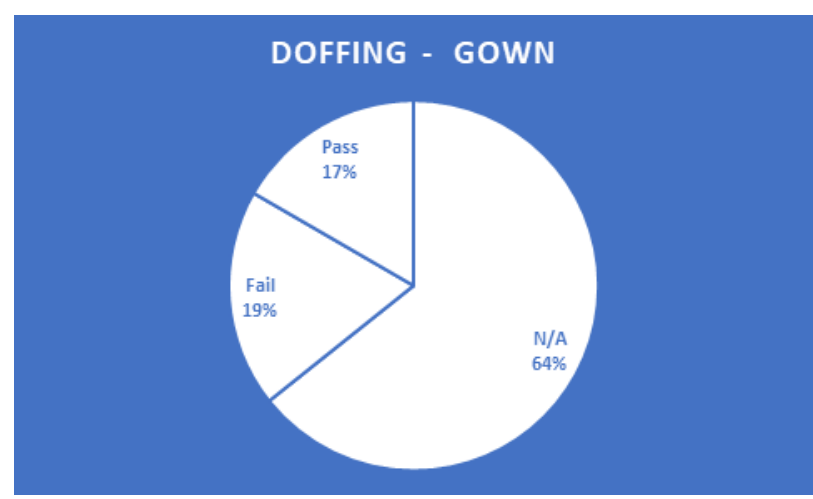

Figure 3. In the doffing part of Gown, seen that $64 \%$ did not perform the skill when needed, $17 \%$ successful demonstrated proper technique and $19 \%$ failed to preform proper technique.

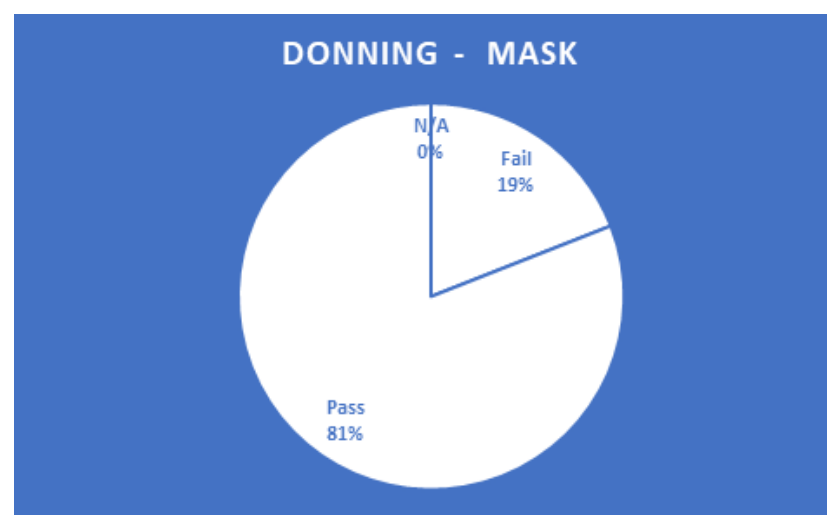

Figure 4. In this segment of Donning - Mask, seen that $81 \%$ successful demonstrated proper technique and $19 \%$ failed to perform proper technique.

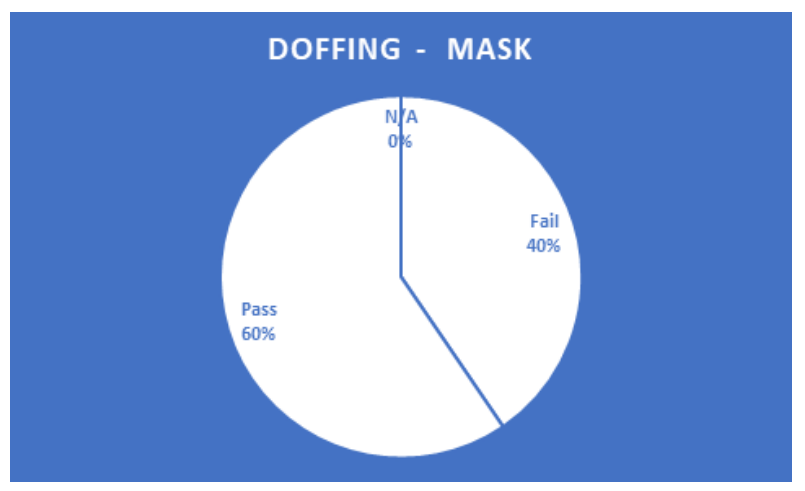

Figure 5. In this segment of Doffing - Mask, seen that $60 \%$ successful demonstrated proper technique and $40 \%$ failed to perform proper technique.

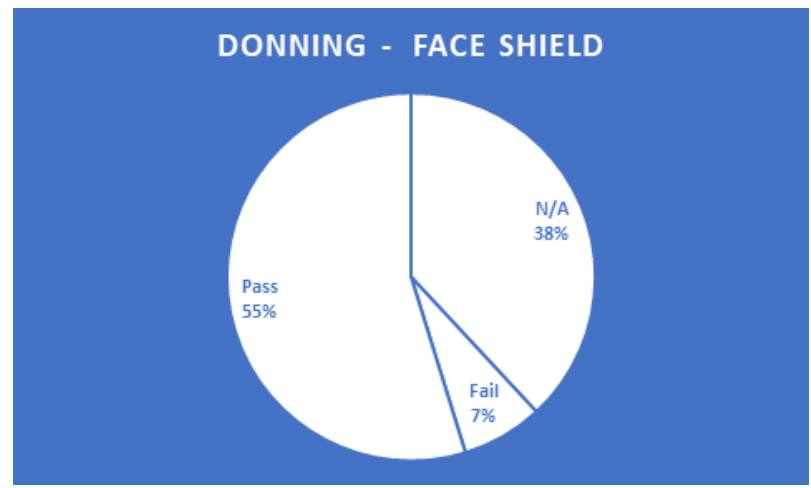

Figure 6. In this segment of Donning - Face Shield, seen that $38 \%$ did not perform the skill when needed, 55\% successful demonstrated proper technique and $7 \%$ failed to perform proper technique.

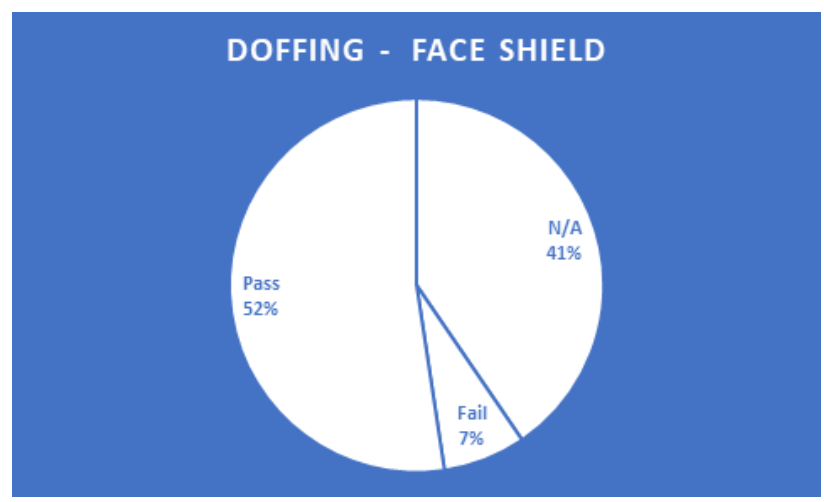

Figure 7. In this segment of Doffing - Face Shield, seen that $41 \%$ did not perform the skill when needed, $52 \%$ successful demonstrated proper technique and $7 \%$ failed to perform proper technique. 


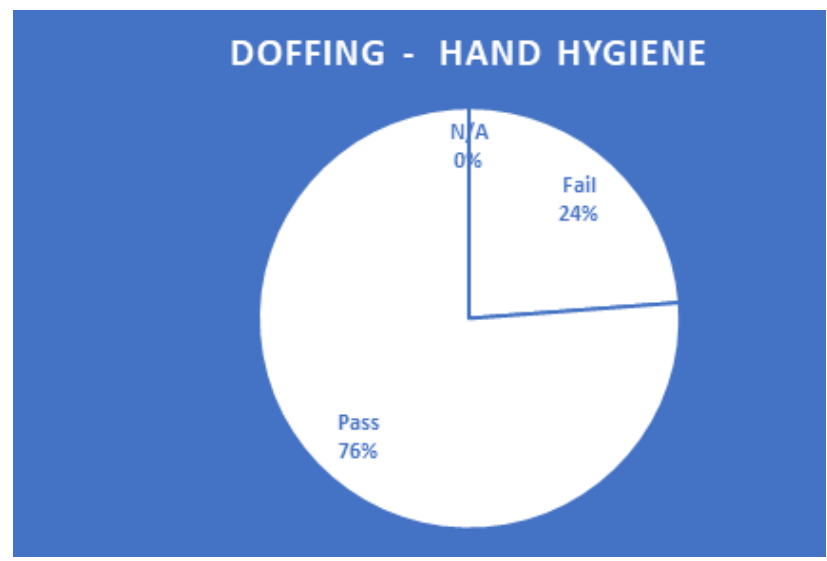

Figure 8. In this segment of Doffing - Hand Hygiene - seen that $76 \%$ successful demonstrated proper technique and $24 \%$ failed to perform proper technique.

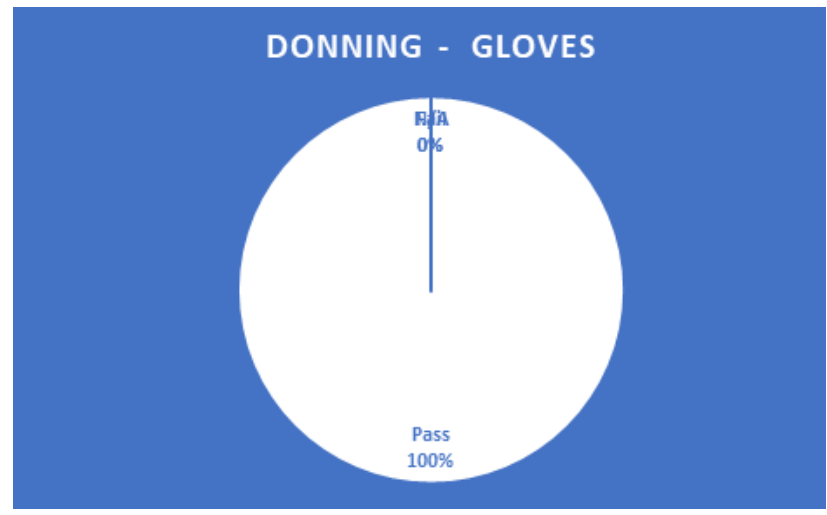

Figure 9. Here there is no fail rate, $100 \%$ successful demonstrated proper technique in Donning - Gloves.

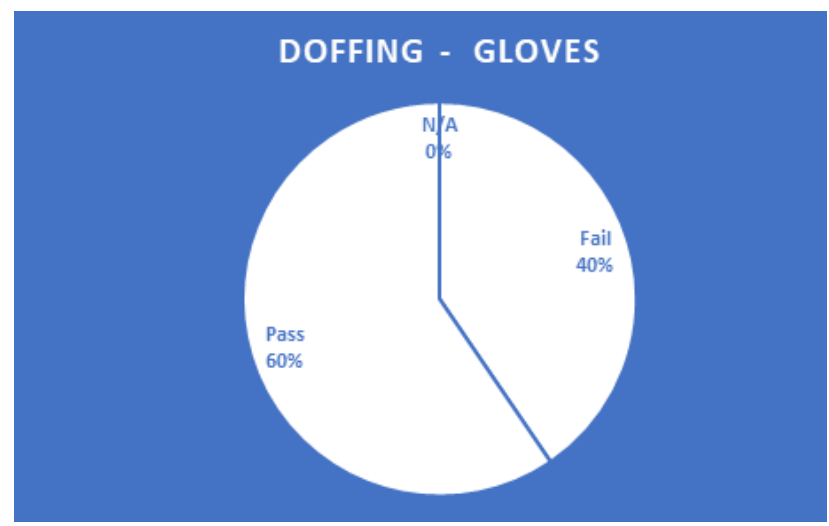

Figure 10. In this segment of Doffing - Gloves, seen that $60 \%$ successful demonstrated proper technique and $40 \%$ failed to perform proper technique.

From the observations, it was clear that the EMS emergency medical team were non-compliant to observe the safety guidelines leading to increased chances of infections.
Additionally, they did not observe the safety procedures regarding PPE, hand hygiene, environmental Decontamination, disinfection, limiting contact with the patient, ambulance cleaning and source control measures aimed at limiting the patient's secretions. The medical team also demonstrated lack of knowledge concerning infection prevention and control standards. In fact, the PPE assessment indicated that most of the emergency medical team as observed in various stations did not follow the standard procedure to fully comply with the standard procedure. Hand hygiene standards, which form critical part of the infection prevention and control criteria, were not complied with. The donning hand hygiene assessments indicated that all the emergency medical staff did not follow the hand sanitizations procedures as required by the guideline's checklist. Similarly, during doffing, a significant number 35 staff members out of $42(83 \%)$ not follow safety guidelines while $4(10 \%)$ not follow the correct procedure and only $3(7 \%)$ followed the right procedure in hand hygiene during doffing process. The indication is that most of the emergency staff were either ignorant of the hand safety procedures or just failed to follow the guidelines.

Non-compliance to follow the standard guidelines was also observable during the other PPE assessments. The PPE procedures that tend to minimize the patient contact including wearing gloves, masks and gowns were not properly followed. The assessment indicates that all the staff followed the correct procedure in donning the gloves. During doffing a significant number $60 \%$ followed the correct procedure while $40 \%$ not follow the right doffing procedure. The increased performance in wearing and putting on gloves could be attributed to the simple procedure involved. However, poor performance was observed in complex procedures. In donning gowns for instance, $67 \%$ of the staff did not implement the required procedure to demonstrate the right technique. Similarly, in doffing the gown, $83 \%$ of the observed staff not follow/perform the skills or follow the correct procedure. Only $17 \%$ demonstrated proper techniques. The other simple PPE procedures such as wearing and removing hair cover were found to be completely ignored by the emergency staff. Similarly, the emergency staff did not implement the shoe cover procedures during donning and doffing processes. Even in procedures they considered important such as wearing face shield and masks, a significant number still did not follow the correct procedures as indicated in the results section. Even though the staff indicated increased techniques in donning and doffing the masks, some of them failed to indicate the correct skills in donning and doffing the masks.

The PPE results further indicated that the emergency staff tend to ignore certain procedures completely such as donning and doffing shoe and hair covers while at the same time failed to implement complex procedures. Even in safety measures considered important such as wearing of gloves a significant number still failed to follow the correct procedure. The failures by the emergency medical staff to follow PPE procedures significantly increase the chances of infections (Haggman et al., 2016). The PPE procedures ensure limited contact with the patient, hand hygiene and environmental cleaning and disinfection control standards.

\section{Discussion}

The study utilized the observation technique as an effective tool in gathering information. This technique was 
useful in measuring the impacts of risky exposure to a worker's general demeanor. The study minimized the impact of interference, since the workers may provide information which is incorrect, by changing their behavior. Saudi Arabia is home to victims of various infectious diseases such as Middle East Respiratory Syndrome MERS. Consequently, these victims may expose other people to these infections. The reaction during transport and treatment of patients deemed risky, was crucial when collecting the data. Although the respondents were informed of the study, during the data collection interference on the worker assignments through questioning or coercion was accepted. The Red Crescent was also supposed to provide permission to gather the data. Information on the respondents was not shared with any other part.

The data collected was collated regarding various types of behaviors observed. The patterns were identified in comparison to treatment of normal patients. Risk related behavior such as withdrawal from patients or willingness to treat patients was one of the expectedbehaviors. Behavior that does not align with the transport and treatment of infectious patients was disregarded.

The data collected from this study is susceptible to various challenges, including workers who do not show their behavior publicly. In addition, the idea of being watched may force the respondents to change their behavior, as such leading to false data being collected. Despite the challenges, the study provided an opportunity for the EMS authority and the medical field at large to learn important lessons. Lessons were on how to improve the quality of care of workers as well as any policies which require improvement in the treatment process.

\section{Recommendations for future training}

From the study, it is clear that EMS do not follow the guidelines and standard procedures to guarantee their safety from infections. In fact, deficiency in following the safety guidelines increases the chances of infections among the health workers. the following are recommended for the EMS.

- Seven days full course training and education program on Standard precautions, Environmental Decontamination and Disinfection procedures to the entire EMS staff. The objective of training and educational program should increase awareness of the safety standards, their applications and compliance in order to reduce infections among the staff. During the training the staff should

- Review the protocols and guidelines of the Saudi Red Crescent Authority

- Roles and responsibilities

- Overview of global response to the pandemic influenza (WorldHealth Organization)

- Identify the risk

- $\quad$ Practicing the PPE

The training and educational program should focus on the work practices that limit the possibilities of infections. The work practices including following the guidelines, ensuring safety environment, PPE and hand washing are key components to comprehensive infection prevention. The main goal of the training program is to maximize the protection against any infection for all the EMS Red Crescent Authority in Saudi Arabia. Even though the training will mainly focus on the personal protective equipment utilization procedures and standards, the broader aim is to sensitize the EMS Red Crescent workforce on the procedures that serve to minimize exposure such as cleaning equipment or areas that have been contaminated, the application of barrier techniques to reduce contamination with the patient's fluids, excreta and secretions.

- The Red Crescent in Saudi Arabia should form Disinfection Control Team (DCT) among the EMS staff specializing on disinfection and control. Since the EMS (Red Crescent) staff in most cases does not have enough time to conduct all the disinfection procedures particularly on the used ambulances, DCT should conduct all the cleaning and disinfection of not only the ambulances but also the used equipment.

- The Red Crescent in Saudi Arabia in collaboration with the Ministry of Interior should also form Environmental Decontamination course within its staff that responds to the environmental decontamination. Decontamination of victims at a scene such aschemical, biological, radiological, or nuclear event is the responsibility of responding Ministry of Interior. Red Crescent should, however, understand basic decontamination procedures in the event decontamination teams are not present and available.

- This includes decontamination procedures for both people and equipment. Even though the specialized team, DCT and Environmental Decontamination Course have never been part of the Saudi Arabia EMS (Red Crescent) staff, it is critical to form them. In fact, apart from saving time, this specialized team enhances coordination of all the work processes in particular following the standard procedures to reduce infections not only to the workforce but also the patients. Besides, there is greater reduction in the workload among the staff since each staff will be just conduct only procedures within their area of specialization. This increases greater attention to the patients, reduces the staff exhaustion, increase motivation, and most importantly enhances compliance to the standard procedures. Therefore, the EMS (Red Crescent) in Saudi Arabia should consider forming these specialized teams to enhance their efficiency and efforts towards infections control and prevention among its staff.

\section{Training and Education Program}

\section{Objectives}

The objective of training and an educational program should increase awareness of the Standard precautions (proper selection and use of PPE), Environmental decontamination, and Disinfection procedures their applications and compliance in order to reduce infections among the staff and save the patients from the infections duringtransportation. In addition, understand basic decontamination 
procedures in the event decontamination teams are not present and available or if the EMS face any events related to Environmental decontamination.

\section{Duration}

- Seven days full course educational and training program

\section{The Training Program Focus Areas}

- Standard precautions

- Environmental decontamination

- Disinfection procedures

\section{Standard precautions}

All the Red Crescent EMS staff will be trained on the standard precautions. The standard precautions are founded on the principles that all bodily fluids and excretions contain infectious organisms. Having skills in the standard precautions is the basic strategy through which EMS personnel could prevent the infectious agents. The standard precautions are supposed to be applied in all EMS settings. The standard precautions practices include critical areas within the standard guidelines such as hand hygiene, PPE and basic work practice controls. The standard precautions include simple measures that the medical staff should observe in order to avoid direct exposure to infectious conditions. The EMS Red Crescent staff in Saudi Arabia should undergo the one weeks training on the standard precaution areas including PPE, hand hygiene and basic work control standards.

As indicated, one-week full course required to all the EMS workers in Saudi Red Crescent. During this training the staff will review the protocols of Red Crescent authority, and the objective of this course it's to protect the workers from the infectious and others. Besides, in this course, the staff will be reviewing the other guidelines and standards such as that of WHO that have the experience of preventing the pandemic in the world. The course on standard precaution remains critical in providing the required knowledge and information about type of the infection and how such infections are prevented.

\section{Practice of the PPE}

Like all the staff that work in high risk areas, the EMS Red Crescent staff in Saudi Arabia will be required to undertake the PPE special training. The staff should be trained on appropriate selection, application and PPE upkeep. The most important part of the training is the standard procedure and compliance. The data indicated situations where the staff found it difficult to follow the PPE simple procedures. Since PPE is cumbersome to the staff in most occasions, the staff needs to be motivated to comply with the standard procedures in order to protect themselves against any infection. Therefore, training should begin with the need to have PPE, its benefits to the staff and the limitations. The staff needs to understand first the need to protect themselves against infectious conditions, their efforts towards that endeavor, which include not only to eliminate the potential hazard but also to use appropriate PPE where necessary. Next is compliance with the standards. The staff will be taught how to handle the equipment, putting it on, wear it appropriately, how to test for proper fit, how to put it off appropriately to avoid any contamination. Constant exercises should be conducted to ensure the staff understands the correct procedure on donning and doffing the equipment and properly using the equipment to avoid infections. The major outcome of the PPE training is to enable employees have appropriate skills in donning and doffing the PPE.

\section{Basic Work Practice Controls}

After completing the PPE training, the staff will undergo another special training on basic work practice controls. The training on basic work practice controls aims to equip the staff with the skills that would enable them to enhance environmental cleaning and disinfection, limit contact with the patient, improve on ambulance cleaning and disinfection and follow source control measures aimed at limiting the patient's secretions. The staff will be taught the basic work practice control standards ranging from hand washing procedures to appropriate identification and disposition of medical waste based on the set regulations.

\section{Hand Hygiene}

The staff will be trained on all hand washing procedures according to the set international standards. Hand hygiene is an integral part of the infection control and prevention. As such, all the steps in hand washing procedures during donning and doffing must be understood by all the staff. Therefore, WHO hand washing procedures will be reviewed by the staff during this training.

\section{Environmental Decontamination}

The staff will be trained on environmental decontamination procedures including the general principles of decontamination and disinfection procedures. The standards procedures include decontaminating equipment used, ambulances and work stations. The staff should constantly be evaluated to ensure that such procedures are followed.

\section{Disinfection Procedures}

The staff also will be trained on the disinfection procedures in order to reduce the risk of contracting contagious diseases. The staff will be trained on all the most probable diseases preventable through disinfection.

The Proposed Training Schedule/ Curriculum Sunday - Thursday 


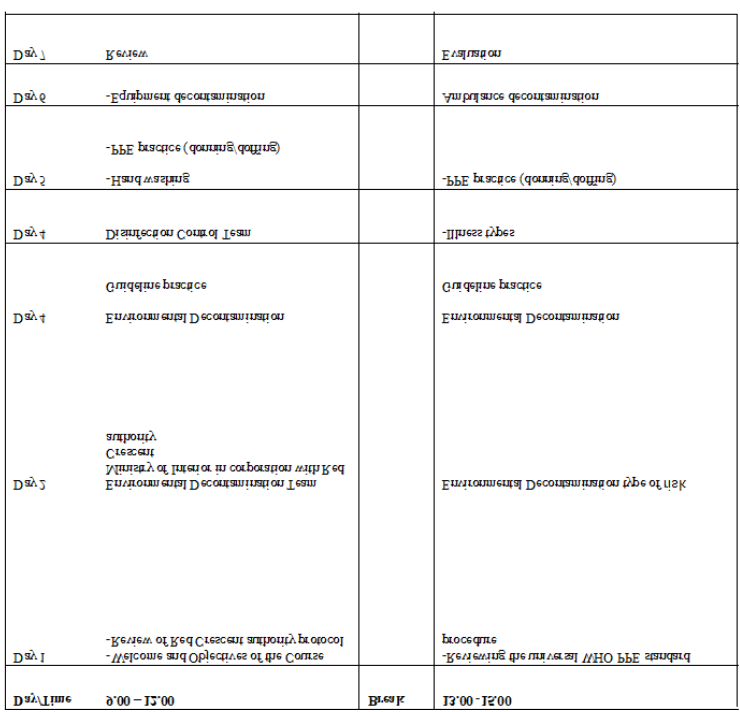

\section{Conclusion}

Lack of following the safety guidelines increases chances of infections from communicable conditions EMS are constantly exposed. A comprehensive training program on the standard guidelines and safety measures to prevent the occurrences of infections is recommended. The training program remains critical in sensitizing the EMS Red Crescent workforce on the procedures that serve to minimize exposure. The Saudi Red Crescent is also encouraged to take measures such as coming up with specialized teams such as DCT in order to further reduce any chances of infections due to lack of time, work related exhaustion among the staff.

\section{References}

1-The National Institute for Occupational Safety and Health (NIOSH). (2017, December 21). 2- Disaster Med Public Health Prep. 2018 Feb 2:1-7. doi: 10.1017/dmp.2017.149. 2-Alnazi, A (2012). Emergency medical services in Saudi Arabia: A study on the significance of paramedics and their experiences on barriers as inhibitors of their efficiency. International Journal of Applied Basic Medicine Research. 2(1):34-7. doi: 10.4103/2229-516X.96803.

3- $\quad$ AlShammari, A, Jennings, $P$ and Williams, B (2017). Evolution of emergency medical services in Saudi Arabia, Journal of Emergency Medicine, Trauma \& Acute
Care 2017:4. http://dx.doi.org/10.5339/jemtac.2017.4 4- $\quad$ Bekefi, T., Epstein M. J., \& Yuthas, K. (2008) Managing opportunities and risks. Ottawa: The Society of Management Accountants of Canada.

5- Coronavirus website- ministry of health, Retrieved January 29, 2018.

6- General Authority for Statistics Real Estate Price Index Fourth Quarter, (January 29, 2018).

7- $\quad$ Gee, S., \& Skovdal, M. (2017) The role of risk perception in willingness to respond to the 2014-2016 West African Ebola outbreak

8- Beltrami, E. M., Williams, I. T., Shapiro, C. N., \& Chamberland, M. E. (2000). Risk and

management of blood-borne infections in health care workers Clinical Microbiology Reviews, 13(3), 385-407.

doi:10.1128/cmr.13.3.385-407.2000

9- $\quad$ Costa, M., Oberholzer-Riss, M., Hatz, C. Steffen, R., Puhan, M., \& Schlagenhauf, P. (2015). Pre- travel health advice guidelines for humanitarian workers: A systematic review. Travel Medicine and Infectious Disease, 13(6), 449-465. doi:10.1016/j.tmaid.2015.11.006 10- Gushulak, B. D. (2017, May 31).

Humanitarian aid workers. Retrieved from https://wwwnc.cdc.gov/travel/yellowbook/2018/advisingtravelers-with-specific-needs/humanitarian-aid-workers 11- Haggman, H., Kenkre, J., \& Wallace, C. (2016). Occupational health for humanitarian aid workers in an Ebola outbreak. Journal of Research in Nursing, 21(1), 2236. doi:10.1177/1744987116630578 12- Koenig, K. L. (2014a). Identify, isolate, inform: A 3-pronged approach to management of public health emergencies. Disaster Medicine and Public Health Preparedness, 9(1), 86-87.

doi:10.1017/dmp.2014.125

13- Koenig, K. L. (2014b). Ebola triage screening and public health: The new "Vital Sign Zero". Disaster Medicine and Public Health Preparedness, 9(1), 57-58. doi:10.1017/dmp.2014.120

14- Reed, E., daya, M. R., Jui, J., Grellman, K., Gerber, L., \& Loveless, M. O. (1993). Occupational infectious disease exposures in EMS personnel. The Journal of Emergency Medicine, 11(1), 9- 16. doi:10.1016/07364679(93)90003-p

15- Beltrami1, E. M., Williams2, I. T., \&

Shapiro2, C. N. (2000, July 01). Elise M. Beltrami. Retrieved April 01, 2018. 